\title{
Effects of Atmospheric Refraction on an Airborne Weather Radar Detection and Correction Method
}

\author{
Lei Wang, ${ }^{1,2}$ Ming Wei, ${ }^{1,3}$ Tao Yang, ${ }^{2}$ and Ping Liu ${ }^{2}$ \\ ${ }^{1}$ Collaborative Innovation Center on Forecast and Evaluation of Meteorological Disasters, NUIST, Nanjing 210044, China \\ ${ }^{2}$ Atmospheric Sounding Technology Center in Sichuan Province, Chengdu 610072, China \\ ${ }^{3}$ State Key Laboratory of Severe Weather, Chinese Academy of Meteorological Sciences, Beijing 100081, China
}

Correspondence should be addressed to Ming Wei; mingweicn123@yeah.net

Received 9 March 2015; Accepted 28 May 2015

Academic Editor: Enrico Ferrero

Copyright (C) 2015 Lei Wang et al. This is an open access article distributed under the Creative Commons Attribution License, which permits unrestricted use, distribution, and reproduction in any medium, provided the original work is properly cited.

\begin{abstract}
This study investigates the effect of atmospheric refraction, affected by temperature, atmospheric pressure, and humidity, on airborne weather radar beam paths. Using three types of typical atmospheric background sounding data, we established a simulation model for an actual transmission path and a fitted correction path of an airborne weather radar beam during airplane take-offs and landings based on initial flight parameters and X-band airborne phased-array weather radar parameters. Errors in an ideal electromagnetic beam propagation path are much greater than those of a fitted path when atmospheric refraction is not considered. The rates of change in the atmospheric refraction index differ with weather conditions and the radar detection angles differ during airplane take-off and landing. Therefore, the airborne radar detection path must be revised in real time according to the specific sounding data and flight parameters. However, an error analysis indicates that a direct linear-fitting method produces significant errors in a negatively refractive atmosphere; a piecewise-fitting method can be adopted to revise the paths according to the actual atmospheric structure. This study provides researchers and practitioners in the aeronautics and astronautics field with updated information regarding the effect of atmospheric refraction on airborne weather radar detection and correction methods.
\end{abstract}

\section{Introduction}

X-band airborne phased-array weather radar relies on computers that control phase changes in array antenna units so that the radar-beam pointing angle can change quickly. This type of radar can obtain targeted weather information in a vertical direction ahead of the airplane in a very short amount of time. Because of the small size and rather high temporal and spatial resolutions of X-band radar, it is particularly suitable for airplanes; it can detect weather in real-time and provide early warnings of hazardous weather conditions at precise locations. The propagation path of the electromagnetic wave emitted by radar bends because of the atmosphere's nonuniform density; this bending is called atmospheric refraction and is common when traditional ground-based radar detects precipitation $[1,2]$. Satelliteborne or airborne weather radar beams also bend when transmitted in the atmosphere because of the uneven distribution of temperature, atmospheric pressure, and vapour [3].
Deng and Liu [4] and Wang and Zhou [5] noted the impact of the ocean environment on airborne radar detection and proposed a correction method for radar detection error. However, these authors did not consider the path-correction method for multiple flight observation angles. Zhang et al. used actual sounding data to analyse the impact of atmospheric refraction on radar-based quantitative precipitation measurements [6]. However, the authors did not describe how to use sounding data to revise the propagation path of an airborne radar beam. Dockery and Kuttler [7] and Yang and Guo used a discrete Fourier transform to analyse, in detail, the propagation characteristics of an electromagnetic wave in an atmospheric duct [8]. Jiang and Wang used a ray-tracing method to correct the atmospheric refraction propagation of satellite-borne radar [9]. However, these algorithms are based on a simple 12-type classification of the atmosphere and do not consider actual atmospheric variations. Therefore, they fail to provide real-time, accurate corrections of the airborne radar detection path. Given that complex and variable sea and 
land surfaces also influence the distributions of temperature, atmospheric pressure, and vapour content in the atmosphere [10], further refraction correction is needed to meet the demands of high-altitude real-time airborne radar [11].

Based on these aforementioned studies, this study aimed at exploring the correction method for the propagation path of the airborne radar beams in different atmospheric backgrounds. Three types of typical atmospheric background sounding data were used to derive the fit for the rate of change in an atmospheric refraction index based on X-band airborne phased-array weather radar parameters and initial flight parameters. Using airborne-radar computer simulation technology $[12,13]$, we established a simulated US standard atmosphere path, an actual propagation path, and a fitted correction path of the radar beam's propagation in the atmosphere during the take-off and landing of an airplane. The method proposed in this study can enhance the precision of radar detection paths and the timely warning of dangerous weather patterns to guarantee flight safety.

\section{Refraction of Ultrashort Waves in the Atmosphere}

According to the law of light refraction,

$$
\frac{\sin i}{\sin r}=\frac{v_{i}}{v_{r}}=\frac{n_{r}}{n_{i}} .
$$

The incidence angle $i$ and refraction angle $r$ of the electromagnetic wave are directly proportional to the propagation velocities $v_{i}, v_{r}$ in different media and are inversely proportional to the refractive exponents $n_{i}, n_{r}$ in different media.

The refraction index is defined as

$$
n=\frac{c}{v}=\frac{\sqrt{\varepsilon^{\prime} \mu^{\prime}}}{\sqrt{\varepsilon_{0} \mu_{0}}}=\sqrt{\frac{\varepsilon^{\prime}}{\varepsilon_{0}}} \sqrt{\frac{\mu^{\prime}}{\mu_{0}}}=\sqrt{\varepsilon \mu} \approx \sqrt{\varepsilon} \quad \varepsilon=1+\chi_{e} .
$$

In (2), $\varepsilon$ is the relative dielectric constant and $\chi_{e}$ is the medium polarisability. $\chi_{e}$ is closely related to the densities of vapour and air molecules in the atmosphere, whereas the densities of vapour and air molecules are related to temperature, humidity, and atmospheric pressure. According to the equation of state for an ideal gas, the atmospheric medium polarisation rate is defined as

$$
\chi_{e}=\varepsilon-1=\frac{a p}{T}+\frac{b e}{T^{2}} .
$$

In (3), $T, e$, and $p$ represent the atmospheric temperature (absolute temperature), the vapour pressure, and the atmospheric pressure, respectively. When combining (2) and (3), it is known that when the radar's electromagnetic wave propagates in the atmosphere, its refraction index $N$ adopts the following relation with $T, e$, and $p$ :

$$
N=(n-1) * 10^{6}=\frac{77.6}{T(h)}\left(p(h)+\frac{4810 e(h)}{T(h)}\right) .
$$

In (4), when the radar's electromagnetic wave propagates through the atmosphere, the atmospheric refractivity is related to temperature, atmospheric pressure, and humidity; the changes in these physical quantities are relevant to the detection height; that is,

$$
\begin{aligned}
\frac{d N}{d h} & =\frac{\partial N}{\partial p} \frac{d p}{d h}+\frac{\partial N}{\partial T} \frac{d T}{d h}+\frac{\partial N}{\partial e} \frac{d e}{d h} \\
& =\frac{A}{T} \frac{d p}{d h}-\frac{A}{T^{2}}\left(p+\frac{2 B e}{T}\right) \frac{d T}{d h}+\frac{A B}{T^{2}} \frac{d e}{d h}
\end{aligned}
$$

In (5), $A$ is 77.6 and $B$ is 4810 [6].

Based on the above equation, it can be seen that the two important parameters that influence the change in the atmospheric refractivity are temperature and humidity (vapour pressure); the atmospheric pressure is a major factor that influences the atmospheric refraction index. During the summer in the low-latitude coastal areas of China, dry warm air moves over a relatively cold sea surface to form a characteristic inversion layer; that is, the temperature increases with altitude and the humidity rapidly decreases with altitude. Because of this, the atmospheric refraction index rapidly decreases with altitude; this process is referred to as atmospheric super-refraction. Conversely, a vertical atmospheric structure forms in the relatively dry midsummer areas of China (e.g., the Loess Plateau) in which the humidity increases with altitude and the temperature decreases with height during weak winds. Because of this, the atmospheric refraction index increases with altitude; this is referred to as negative refraction. The effects of these typical weather conditions on the changes in the atmospheric refraction index are analysed in detail in the following section.

\section{Correction Method for Airborne Radar Beam Paths}

As noted in the preceding section, when an electromagnetic radar beam propagates through the atmosphere, it bends due to the changes in the refractivity at different altitudes. When an airborne radar system detects hazardous weather conditions, the real-time change in the target's distance from the airplane is a major concern. A two-dimensional Cartesian coordinate system was established to calculate this change. The $x$-coordinate represented the horizontal distance of the target from the airplane, and the $y$-coordinate represented the vertical height of the target from the airplane. The propagation path of the electromagnetic wave was $r$. The coordinate origin indicated the airplane's current position, and the initial refractivity of the airplane's height was $N_{h 0}$. The airborne radar beam performed a vertical electrical scan with an initial pitch angle of $\theta_{0}$ (the negative value represents the angle of depression, while the positive value represents the angle of elevation). The following equation was then applied:

$$
\begin{aligned}
& \frac{d x}{d h}=\operatorname{tg} \theta_{h}, \\
& \frac{d h}{d r}=\cos \theta_{h} .
\end{aligned}
$$


In (1) and (5), the relation between the angle of pitch $\theta_{h}$ and the random vertical distance from the airplane was

$$
\sin \left(90-\theta_{h}\right)=\frac{N_{h 0} \sin \left(90-\theta_{0}\right)}{N_{h 0}+(h-h 0) *\left((A / T)(d p / d h)-\left(A / T^{2}\right)(p+2 B e / T)(d T / d h)+\left(A B / T^{2}\right)(d e / d h)\right)} .
$$

According to (7), it is only necessary to know the background information on the present state of the atmosphere to estimate the refraction angles at different altitudes; the actual distance of the target from the location can be estimated.

When an airborne weather radar system detects a weather target, its transmitted electromagnetic beam will bend in the atmosphere due to the uneven vertical distribution of atmospheric refractivity. Atmospheric refractivity changes with temperature, atmospheric pressure, and humidity in the vertical direction. The correction methods for the actual propagation paths of an airborne radar beam under three types of weather conditions are provided in this section.

The initial flight parameters and radar parameters are shown in Table 1. In this table, the initial flight height is measured from sea level. The electromagnetic wave propagation paths of the airborne weather radar were revised according to three typical weather conditions.

(A) Standard atmosphere. The US standard atmospheric parameters are shown in Table 2.

Based on the parameters in Tables 1 and 2, a numerical method was adopted to derive solutions for (6) and (7). Assuming that the airplane landed from an initial height of 1,500 metres and that the airborne radar performed an overlooking vertical scan, the values of the depression angles were $5^{\circ}$ and $30^{\circ}$, and the independent variable of the vertical distance $h$ was $-150 \mathrm{~m}$ :

$$
\begin{aligned}
\frac{d N}{d h} & =-4 \times 10^{-2} \mathrm{~m}^{-1}, \\
\sin \left(90-\theta_{h+1}\right) & =\frac{N_{h} * \sin \left(90-\theta_{h}\right)}{N_{h}-150 \times(d N / d h)}, \\
& \text { height }=1500, N_{h 0}=261, \theta_{0}=5,30, \\
\operatorname{dis}_{h+1} & =\operatorname{dis}_{h}+150 \times \operatorname{tg} \theta_{h+1}, \quad \operatorname{dis}_{h 0}=0, \\
\operatorname{radial}_{h+1} & =\operatorname{radial}_{h}+\frac{150}{\cos \theta_{h+1}}, \quad \operatorname{radial}_{h 0}=0 .
\end{aligned}
$$

Based on the parameters in Tables 1 and 2, a numerical method was adopted to derive solutions for (6) and (7). Assuming that the airplane took off at an initial height of 0 metres and that the airborne radar performed an upward vertical scan, the values of the depression angles were $10^{\circ}$ and $30^{\circ}$, and the independent variable of the vertical distance $h$ was $150 \mathrm{~m}$ :

$$
\begin{aligned}
\frac{d N}{d h} & =-4 \times 10^{-2} \mathrm{~m}^{-1}, \\
\sin \left(90-\theta_{h+1}\right) & =\frac{N_{h} * \sin \left(90-\theta_{h}\right)}{N_{h}+150 \times(d N / d h)}, \\
& \text { height }=0, N_{h 0}=318, \theta_{0}=10,30,
\end{aligned}
$$

$$
\begin{aligned}
\operatorname{dis}_{h+1} & =\operatorname{dis}_{h}+150 \times \operatorname{tg} \theta_{h+1}, \quad \operatorname{dis}_{h 0}=0, \\
\operatorname{radial}_{h+1} & =\operatorname{radial}_{h}+\frac{150}{\cos \theta_{h+1}}, \quad \operatorname{radial}_{h 0}=0 .
\end{aligned}
$$

In (9), total reflection occurred when $\sin \theta_{h+1}>1$. At this moment, the electromagnetic beam bent downward and was then refracted and transmitted; the detection height also decreased. When the electromagnetic beam was transmitted to the ground, it also reflected off of the ground. Afterwards, the beam bent upward and was then refracted and transmitted. This entire process continued repeatedly. By using (8) and (9), the actual propagation path of the airborne radar beam could be obtained for the airplane's take-off and landing, as shown in Figures 4 and 5.

(B) The actual distributions of the temperature, atmospheric pressure, and humidity at different heights do not necessarily follow the distribution law of the standard atmosphere; therefore, it is necessary to make real-time calculation adjustments according to the sounding data of the present flight area. The sounding data of the Haikou area at 8:00, August 2, 2014, are shown in Table 3.

The water vapour pressure data in Table 3 was obtained based on the Magnus Empirical Formula [14]:

$$
e=f \times 6.1078 \times \exp \left(\frac{17.269 \times(T-273.16)}{T-35.86}\right) .
$$

In (10), $f$ represents the relative humidity, $T$ the absolute temperature, and $e$ the actual vapour pressure (unit: $\mathrm{hPa}$ ). Equation (10) and observation data such as temperature and atmospheric pressure were inserted into (4) to obtain the refraction index $N$ under the weather conditions. A linear fit was applied to the height values in the table; the refraction index $N$ corresponds to the heights obtained through the calculation. The change rate of the approximate refraction index can be obtained under the background weather conditions, as indicated in Figure 1.

The slope of the linear fit was -0.0422 ; that is, the change rate of the refraction index at this time was

$$
\frac{d N}{d h}=-4.22 \times 10^{-2} \mathrm{~m}^{-1} .
$$

In (11), the change rate of the refraction index under these atmospheric conditions was similar to that of the standard atmosphere; the propagation path of the airborne radar beam was similar to that of the standard atmosphere. Equation (11) was inserted into (8) and (9) in order to obtain the position detected by the airborne radar beam at that moment.

(C) The data for one sounding in the Taiyuan area on July 6,2014 , is shown in Table 4 . The refraction index was obtained 
TABLE 1: Initial flight parameters and radar parameters.

\begin{tabular}{lcccl}
\hline Name of parameters & Unit & Initial value & Scope & Meaning \\
\hline Height & $\mathrm{m}$ & 1500 & $0 \sim 1500$ & $\begin{array}{l}\text { Initial descending height } \\
\text { Initial take-off height }\end{array}$ \\
$h$ & $\mathrm{~m}$ & 0 & $0 \sim 1500$ & $\begin{array}{l}\text { Vertical distance of the target from the airplane } \\
\text { as the airplane changes altitude }\end{array}$ \\
$\alpha$ & $\circ$ & 0 & $0 \sim 359$ & Flight course \\
$\theta_{h}$ & $\circ$ & 60 & $-60 \sim 60$ & Pitch angle of vertical electrical scan \\
Dis & $\mathrm{m}$ & $5,10,30$ & $0 \sim 20000$ & Horizontal distance from the plane \\
radial & $\mathrm{m}$ & 0 & $0 \sim 20000$ & $\begin{array}{l}\text { Actual radial distance of electromagnetic wave } \\
\text { beam } \\
f\end{array}$ \\
$\mu$ & $\mathrm{GHz}$ & 0.3 & Radar frequency \\
$\mu$ & $\mu s$ & 1 & Radar pulse width \\
PRF & $\mathrm{Hz}$ & 1736 & Pulse repetition frequency
\end{tabular}

TABLE 2: Standard atmospheric parameters.

\begin{tabular}{lcccc}
\hline Altitude $(\mathrm{m})$ & $\begin{array}{c}\text { Temperature } \\
\left({ }^{\circ} \mathrm{C}\right)\end{array}$ & $\begin{array}{c}\text { Atmospheric pressure } \\
(\mathrm{hPa})\end{array}$ & $\begin{array}{c}\text { Vapour pressure } \\
(\mathrm{hPa})\end{array}$ & Refraction index \\
\hline 0 & 15.0 & 1013 & 10.2 & 318 \\
150 & 14.0 & 995 & 9.6 & 312 \\
300 & 13.0 & 977 & 9.0 & 306 \\
500 & 11.0 & 955 & 8.3 & 299 \\
1000 & 8.5 & 894 & 6.7 & 278 \\
1500 & 5.2 & 845 & 5.3 & 261 \\
\hline
\end{tabular}

TABLE 3: Sounding data for the Haikou area at 8:00, August 2, 2014.

\begin{tabular}{lcccc}
\hline Altitude $(\mathrm{m})$ & $\begin{array}{c}\text { Temperature } \\
\left({ }^{\circ} \mathrm{C}\right)\end{array}$ & $\begin{array}{c}\text { Atmospheric pressure } \\
(\mathrm{hPa})\end{array}$ & $\begin{array}{c}\text { Vapour pressure } \\
(\mathrm{hPa})\end{array}$ & Refraction index \\
\hline 24 & 27.8 & 998 & 27.6 & 371.43 \\
157 & 27.0 & 984 & 27.1 & 366.82 \\
401 & 29.4 & 959 & 22.5 & 338.05 \\
550 & 28.4 & 944 & 22.8 & 336.82 \\
742 & 27.0 & 925 & 23.5 & 336.79 \\
876 & 26.2 & 911 & 22.4 & 329.81 \\
1098 & 24.9 & 893 & 21.1 & 321.29 \\
1375 & 23.0 & 876 & 19.4 & 312.18 \\
1486 & 22.6 & 850 & 17.8 & 299.22 \\
\hline
\end{tabular}

TABLE 4: Sounding data at 8:00 on July 6, 2014, for the Taiyuan area.

\begin{tabular}{lcccc}
\hline Altitude $(\mathrm{m})$ & $\begin{array}{c}\text { Temperature } \\
\left({ }^{\circ} \mathrm{C}\right)\end{array}$ & $\begin{array}{c}\text { Atmospheric pressure } \\
(\mathrm{hPa})\end{array}$ & $\begin{array}{c}\text { Vapour pressure } \\
(\mathrm{hPa})\end{array}$ & Refraction index \\
\hline 779 & 20.1 & 921 & 15.3 & 310.24 \\
1079 & 19.4 & 895 & 16.4 & 309.27 \\
1293 & 18.9 & 868 & 18.8 & 312.96 \\
1390 & 18.3 & 859 & 18.5 & 310.24 \\
1475 & 17.6 & 850 & 18.9 & 310.54 \\
1607 & 17.2 & 837 & 18.6 & 306.38 \\
\hline
\end{tabular}




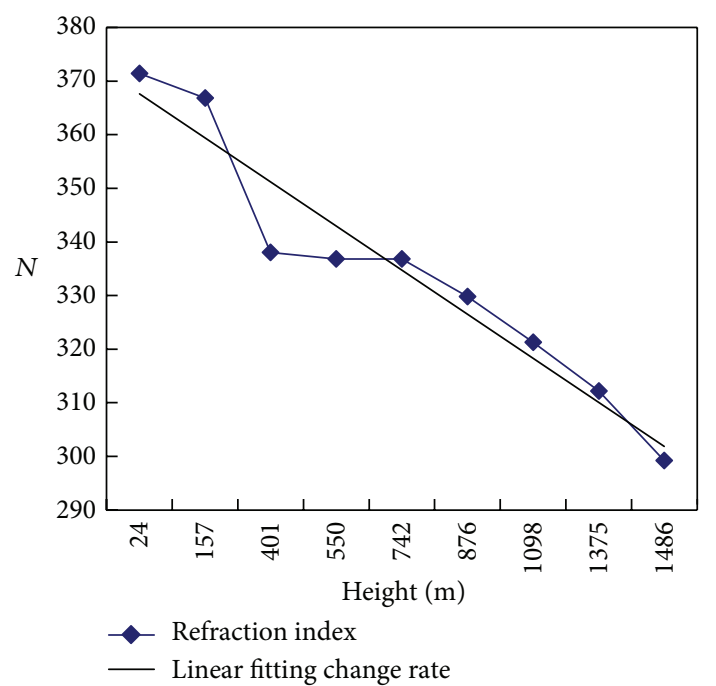

Figure 1: Linear-fit change rate of the refraction index $(N)$ under (B) conditions.

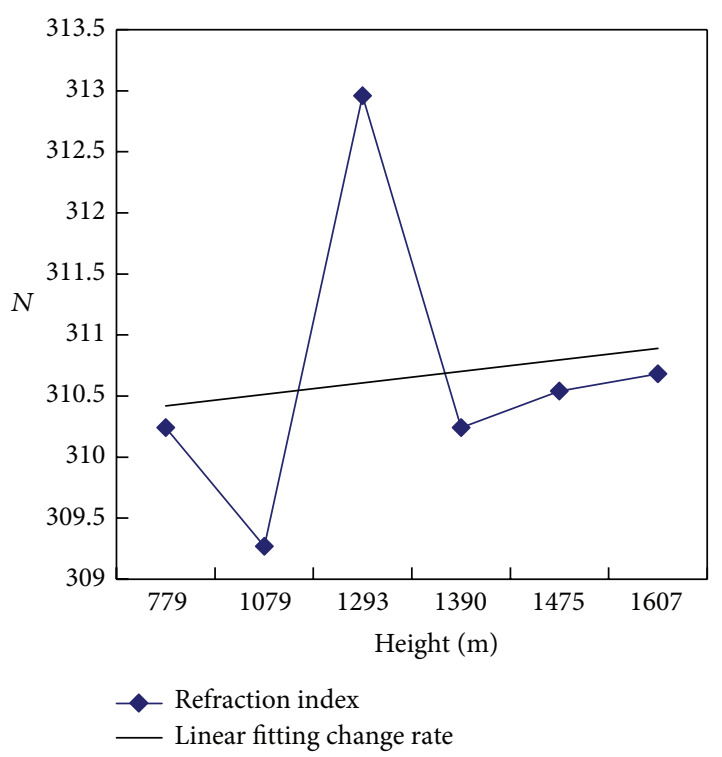

Figure 2: Change rate of the linear fit of the refraction index $(N)$ under (C) conditions.

for different heights using the method in Part (B) and a linear fit was applied; as a result, the change rate of the refraction index could be obtained under particular weather conditions, as indicated in Figure 2.

The slope of the linear fit was 0.0053 , and the change rate of the refraction index at this moment was determined by the following equation:

$$
\frac{d N}{d h}=0.53 \times 10^{-2} \mathrm{~m}^{-1}
$$

In (12), the refraction index increased with height under the specific weather conditions, in contrast to the previous two circumstances. According to the parameters in Figures 1 and 4, the numerical value method was adopted to derive

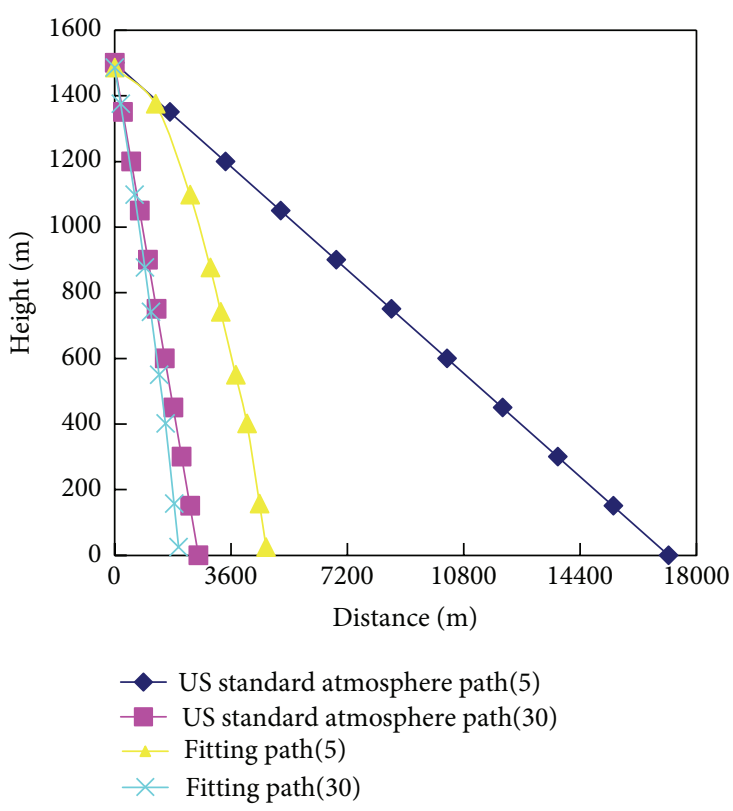

FIGURE 3: The propagation path of the radar beam in a superrefractive atmosphere during the airplane's landing.
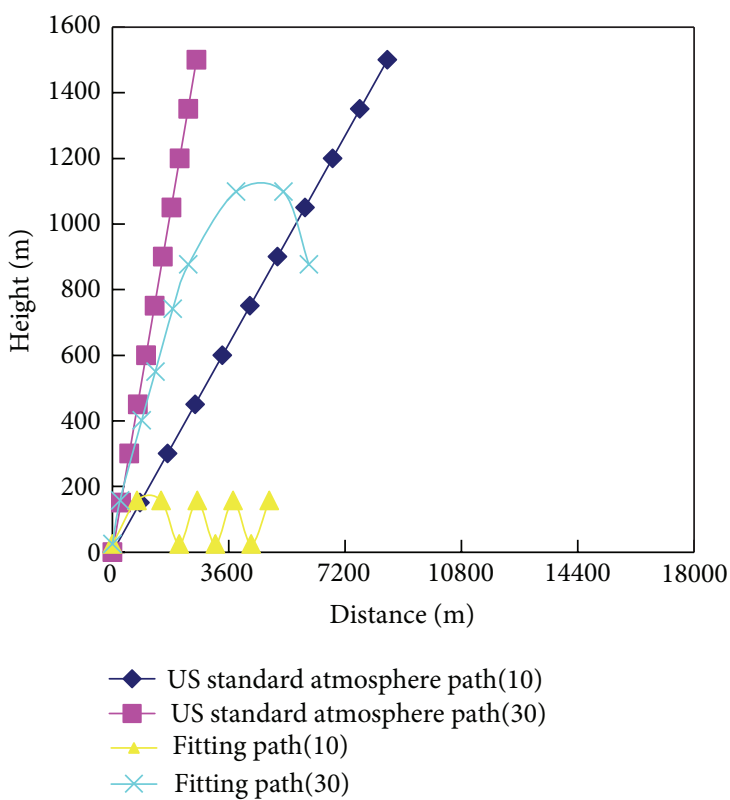

FIgURE 4: The propagation path of the radar beam in a superrefractive atmosphere during the airplane's take-off.

solutions for (6) and (7). Assuming that the airplane was landing from an initial height of 1,500 metres and that the airborne radar conducted an overlooking vertical scan, the depression angles were $10^{\circ}$ and $30^{\circ}$, and the independent variable of the vertical distance $h$ was $-150 \mathrm{~m}$ :

$$
\begin{gathered}
\frac{d N}{d h}=0.53 \times 10^{-2} \mathrm{~m}^{-1}, \\
\sin \left(90-\theta_{h+1}\right)=\frac{N_{h} * \sin \left(90-\theta_{h}\right)}{N_{h}-150 \times(d N / d h)}, \\
\text { height }=1500, N_{h 0}=261, \theta_{0}=10,30,
\end{gathered}
$$




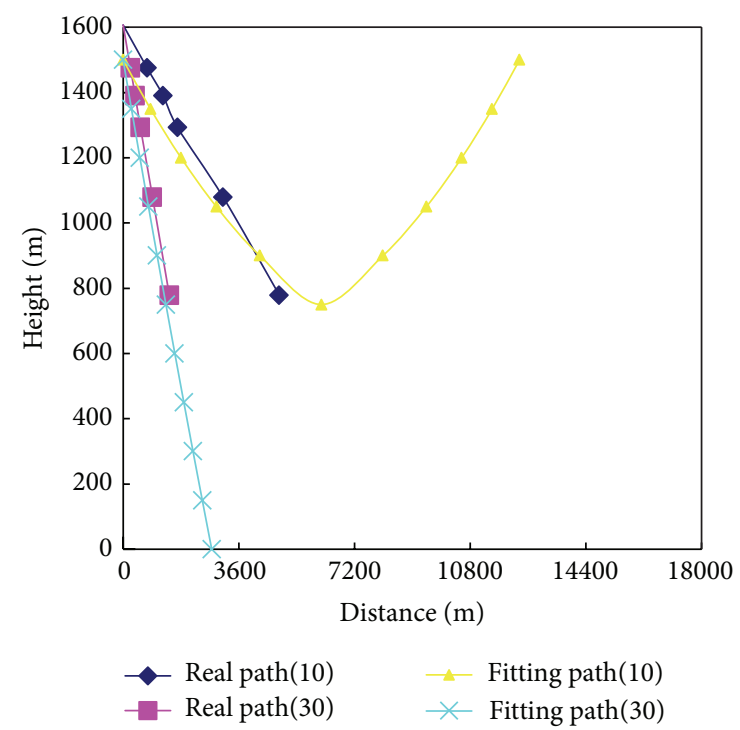

FIgURE 5: The propagation path of the radar beam $i$ in a negative refraction atmosphere during the airplane's landing.

$$
\begin{gathered}
\operatorname{dis}_{h+1}=\operatorname{dis}_{h}+150 \times \operatorname{tg} \theta_{h+1}, \quad \operatorname{dis}_{h 0}=0, \\
\operatorname{radial}_{h+1}=\operatorname{radial}_{h}+\frac{150}{\cos \theta_{h+1}}, \quad \operatorname{rdial}_{h 0}=0 .
\end{gathered}
$$

In (13), total reflection would occur when $\sin \theta_{h+1}>1$. At this moment, the airborne beam would bend upward and transmit while the detection height would decrease. Based on (13), the actual propagation path of the radar beam could be obtained when the airplane was landing in these specific weather conditions.

\section{Simulation Results and Analysis}

Based on the correction methods for the airborne radar beam paths provided in Section 3, the actual propagation paths of the electromagnetic radar beam under different weather conditions were obtained. The propagation path of the radar beam is shown in Figure 3 for when the airplane was landing in a super-refractive atmosphere. The propagation path of the electromagnetic wave is shown in Figure 4 in a super-refractive atmosphere during the airplane's take-off. The propagation path of the radar beam is shown in Figure 5 in a negative refraction atmosphere during the airplane's landing.

In Figure 3, the propagation path of the airborne radar beam without considering refraction was linear under the super-refractive atmospheric condition, whereas the propagation path of the airborne radar beam when considering refraction had a downward bending shape. This difference is primarily due to $d N / d h<0$ when the airborne radar operated at an angle of depression; in addition, the electromagnetic beam was always transmitted from a thin medium to a thick medium. In Figure 3, the airplane height was 1,500 metres. When the airplane was ready to land, the airborne radar conducted a vertical downward electrical scan. With a depression angle of $5^{\circ}$, the horizontal distance of the electromagnetic beam approaching the ground without considering refraction was approximately 17 kilometres, while the horizontal distance of the electromagnetic beam approaching the ground while considering refraction was 5.1 kilometres. This constitutes a significant error between the detected positions, and it was necessary to correct the error. When the depression angle increased to $30^{\circ}$, the horizontal distance of the electromagnetic beam approaching the ground without considering refraction was approximately 3.2 kilometres, while the horizontal distance when considering refraction was 2.5 kilometres. The error between these distances was relatively small, indicating that an increase in the depression angle can decrease the detected distance error caused by the standard atmospheric refraction.

The atmosphere in Figure 4 was identical to that in Figure 3. The airplane had just initiated take-off. The airborne radar was conducting a vertical upward electrical scan, and the electromagnetic beam was constantly transmitted from a thick medium to a thin medium; therefore, total reflection was likely to occur. In Figure 4, the airplane was ready to take off from the ground at an elevation angle of $10^{\circ}$. When the electromagnetic beam was at a vertical distance of one kilometre without considering refraction, the horizontal distance from the airplane was approximately 3.8 kilometres. Conversely, the electromagnetic beam was repeatedly reflected into the ground when refraction was considered; therefore, the beam was unexpectedly unable to detect the target. When the elevation angle was $30^{\circ}$ and the electromagnetic beam without considering refraction detected a vertical distance of 1.5 kilometres, the horizontal distance from the airplane was approximately 3.1 kilometres. In contrast, the electromagnetic beam when considering refraction was unable to reach this detection height; therefore, under this condition, the total reflection must be considered and the detection distance must be corrected. In addition, the elevation angle should be increased to avoid repeated ground reflections caused by super-refraction.

Figure 5 shows that, under typical negatively refractive atmospheric conditions, the electromagnetic beam was constantly transmitted from a thick medium to a thin medium when the airborne radar conducted observations at a downward depression angle because of $d N / d h>0$. The electromagnetic beam was transmitted in a curving upward manner; thus, total refraction was likely to occur. In Figure 5, the airplane height was 1,500 metres. The airplane was ready to land and the airborne weather radar conducted a vertical downward electrical scan. With a depression angle of $10^{\circ}$, the electromagnetic beam with refraction experienced total refraction and could not reach the ground; as a result, the beam bent upward. When the depression angle was $30^{\circ}$, the horizontal distance of the electromagnetic beam approaching the ground when considering refraction was approximately 3.4 kilometres, indicating that an increase in the depression angle can avoid the total reflection caused by the negatively refractive atmosphere and can gradually reduce the detection distance error. 
TABLE 5: Correlation coefficients of the refractivity $(N)$ with height.

\begin{tabular}{lccccc}
\hline \multicolumn{3}{c}{ Haikou } & \multicolumn{3}{c}{ Taiyuan } \\
$\begin{array}{l}\text { Height } \\
(\mathrm{m})\end{array}$ & $N$ & Correlation & $\begin{array}{c}\text { Height } \\
(\mathrm{m})\end{array}$ & $N$ & Correlation \\
\hline 24 & 371.4 & & 779 & 310.2 & \\
401 & 336.5 & & 1079 & 309.3 & \\
742 & 336.5 & -0.96 & 1293 & 312.9 & 0.31 \\
876 & 329.9 & & 1390 & 310.2 & \\
1098 & 321.3 & & 1474 & 310.5 & \\
1375 & 312.2 & & 1607 & 306.4 & \\
\hline
\end{tabular}

Errors also exist between the change in the electromagnetic wave propagation path caused by the actual atmospheric refractivity and the fit path. Table 5 shows that the refractivity changes with the change in the height data. The correlation coefficients in the Haikou area during atmospheric superrefraction and those in the Taiyuan area during atmospheric negative refraction are also shown in Table 5. Under superrefractive atmospheric conditions in the Haikou area, the correlation coefficients of the refractivity with height reached -0.96; that is, a high negative correlation existed; therefore, the error between the electromagnetic propagation path obtained by adopting the linear-fit method and the actual propagation path was relatively small. Under the negatively refractive atmospheric conditions in the Taiyuan area, the correlation coefficient of the refractivity with height was only 0.31 , which is a low positive correlation; therefore, the error between the electromagnetic propagation path obtained by adopting the linear-fit method and the actual propagation path was quite significant. Table 6 shows the error between the actual horizontal distance of the target detected by the radar and the fitted horizontal distance under these two typical atmospheric conditions. As shown in Table 6, the errors in the fitted horizontal distance of the target positions detected by the radar and the actual values were mostly below 0.1 . If the pitch angle increased, the relative error would be further reduced. In combining Table 6 and Figure 5, it can be seen that, under negative atmospheric conditions during the airplane's landing, the errors in the fitted horizontal distance of the target positions detected by the radar and the actual values were significant (mostly above 0.4 ). The simple fitting method ignored the domains of drastic variations in the actual atmosphere. When the radar scanned at a depression angle of $10^{\circ}$, the actual electromagnetic beam did not undergo total reflection, in contrast to the fitting path.

Our analysis demonstrates the real-time variation in the vertical structure of the atmosphere and that the traditional method for the correction of atmospheric refraction based on several typical atmospheric classification models cannot satisfy the demands of real-time radar detection in terms of accuracy. Therefore, to realize the real-time, accurate correction of the airborne radar detection path, a large amount of real-time vertical physical data of the atmosphere generated by advanced detection devices must be obtained.
TABLE 6: Relative error in the electromagnetic wave propagation path during the airplane's landing.

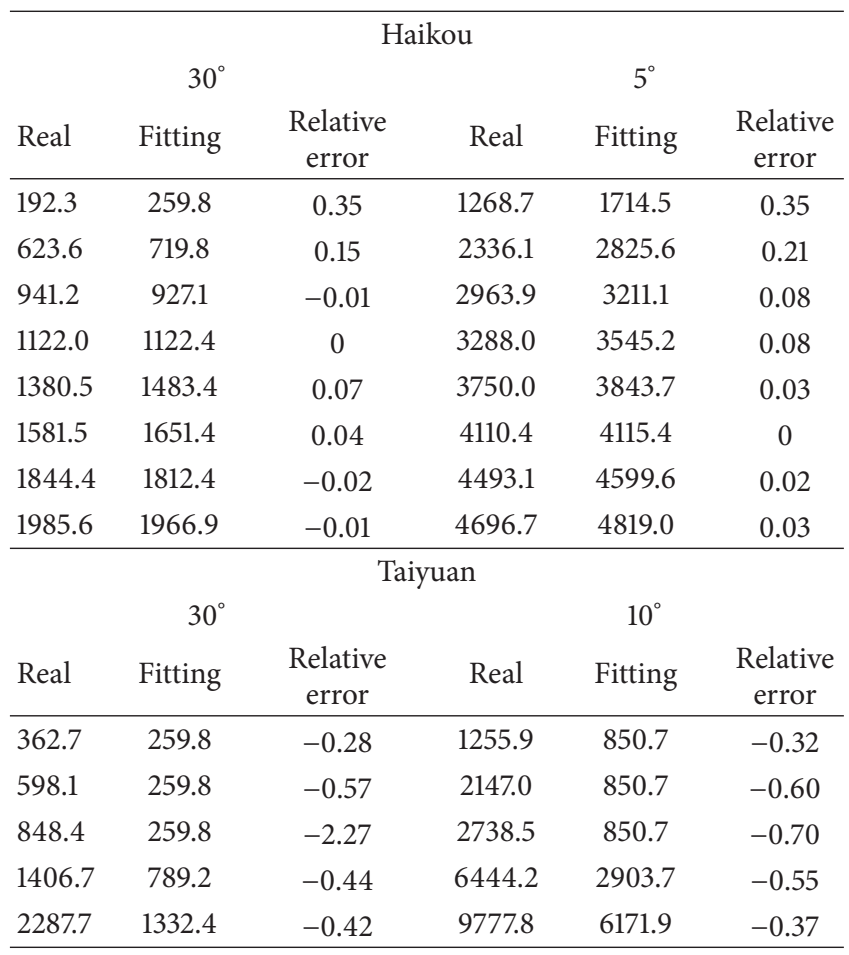

\section{Conclusions}

This study analysed the propagation paths of an airborne weather radar beam in different atmospheric conditions and proposed correction methods. In a super-refractive atmosphere, the airborne radar obtained observations at a depression angle and the electromagnetic beam was always transmitted from a thin medium to a thick medium; therefore, the propagation path of the electromagnetic beam bent downward and did not undergo total reflection. When the airborne radar obtained observations at an elevation angle, the electromagnetic beam was always transmitted from a thick medium to a thin medium; therefore, total reflection would occur, particularly with low elevation angles. The electromagnetic wave was likely to be reflected into the ground and cause repeated refractions that would severely interfere with target detection. Therefore, when the airborne radar is obtaining observations at an elevation angle during airplane take-off, the elevation angle should be increased as much as possible. In a negative-refraction atmosphere, the airborne radar obtained observations at a depression angle and the electromagnetic beam was always transmitted from a thick medium to a thin medium; therefore, total reflection was likely to occur. The electromagnetic beam bent and transmitted upward. When the airborne radar operated at an upward elevation angle, the electromagnetic beam was always transmitted from a thin medium to a thick medium. The propagation path of the electromagnetic beam still bent upward, and it did not undergo total reflection.

During the airplane's take-off and landing, the maximum horizontal detection range of the airborne weather radar 
beam was normally within $30 \mathrm{~km}$. Therefore, the impact of the curvature of the earth was ignored in this study, which reduced computational complexity and increased computational speed. In addition, the fitted-beam path based on the actual sounding data proposed in this study was closer to the actual values than the traditional correction methods. This algorithm is expected to more accurately and quickly provide the actual echo position of the airborne radar and thus more effectively guarantee flight safety.

Under negatively refractive atmospheric conditions, the positive correlation of the refractivity with height is very low; adopting the direct-fitting method will result in significant errors. Traditional radio sounding data can only be gathered four times per day, which cannot reflect the variations in the vertical distribution of the atmosphere in a real-time manner. In the future, real-time data of atmospheric vertical structures can be obtained through global positioning system radio occultation inversion [15], and the piecewise-fitting method can then be adopted to obtain a more accurate atmospheric refraction rate to further increase the correction precision of the electromagnetic wave refraction path. However, this will require the statistical analysis of a large amount of data.

\section{Conflict of Interests}

The authors declare no conflict of interests.

\section{Acknowledgments}

The project was funded by the Key Project of Chinese National Programs for Fundamental Research and Development (973 Program) (2012CB430102), the Aero Science Foundation of China (201320R2001), the Nonprofit Industry (Meteorological) Research Project (GYHY201206038 and GYHY201306040), the Key Technology Project of the State Meteorological Administration in 2014 (CMAGJ2014M21), and the Key Technology Project of the State Meteorological Administration in 2015 (CMAGJ2015M51).

\section{References}

[1] E. E. Altshuler, "Tropospheric range-error corrections for the global positioning system," IEEE Transactions on Antennas and Propagation, vol. 46, no. 5, pp. 643-649, 1998.

[2] G. F. Zhang and R. J. Doviak, "Bistatic interferometry to measure clear air wind," in Proceedings of the 32nd Conference on Radar Meteorology, Albuquerque, NM, USA, 2005.

[3] H. Wang, M. Wei, G. P. Li, S. Zhou, and Q. Zeng, "Analysis of precipitable water vapor from GPS measurements in Chengdu region: distribution and evolution characteristics in autumn," Advances in Space Research, vol. 52, no. 4, pp. 656-667, 2013.

[4] Z. L. Deng and M. L. Liu, "Analysis of ocean environment impact on airborne radar," Ship Electronic Engineering, vol. 30, pp. 89-91, 2010.

[5] W. Y. Wang and X. L. Zhou, "The research of electromagnetic wave characteristic affected by atmospheric refraction index," Science Technology and Engineering, vol. 12, no. 32, pp. 87268729, 2012.
[6] P. C. Zhang, B. Y. Du, and T. P. Dai, Radar Meteorology, Meteorological Press, Beijing, China, 2000.

[7] G. D. Dockery and J. R. Kuttler, "An improved impedanceboundary algorithm for fourier split-step solutions of the parabolic wave equation," IEEE Transactions on Antennas and Propagation, vol. 44, no. 12, pp. 1592-1599, 1996.

[8] C. Yang and L. X. Guo, "Study the propagation characteristic of radio wave in atmospheric duct," Journal of Xidian University, vol. 36, no. 6, pp. 1087-1095, 2009.

[9] C. Jiang and B. Wang, "Atmospheric refraction corrections of radiowave propagation for airborne and satellite-borne radars," Science in China, Series E: Technological Sciences, vol. 44, no. 3, pp. 280-290, 2001.

[10] R. Kevin and T. Walters, "Single doppler observations of boundary layer flows over heterogeneous terrain," in Proceedings of the 32nd Conference on Radar Meteorology, Albuquerque, NM, USA, 2005.

[11] M. Denny, "Refracted propagation effects for airborne radar," in Proceedings of the IEEE International Radar Conference, pp. 554-559, May 2000.

[12] M. Wei and S. J. Zhang, "Simulation algorithm research of an airborne doppler weather with radar scanning ideal model," Transactions of Atmospheric Sciences, vol. 34, no. 1, pp. 28-35, 2011.

[13] Z. X. Gao, H. B. Gu, and H. Liu, "Real-time simulation of large aircraft flying through microburst wind field," Chinese Journal of Aeronautics, vol. 22, no. 5, pp. 459-466, 2009.

[14] P. X. Sheng and J. T. Mao, Atmospheric Physics, Peking University Press, Beijing, China, 2003.

[15] J. S. Haase, B. J. Murphy, P. Muradyan et al., "First results from an airborne GPS radio occultation system for atmospheric profiling," Geophysical Research Letters, vol. 41, no. 5, pp. 17591765, 2014. 

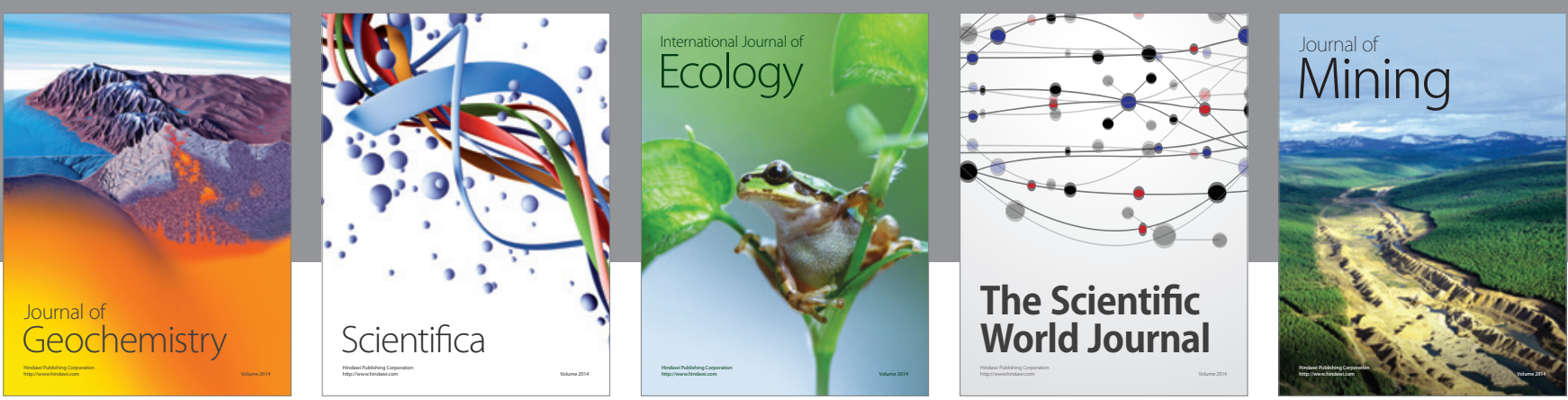

The Scientific World Journal
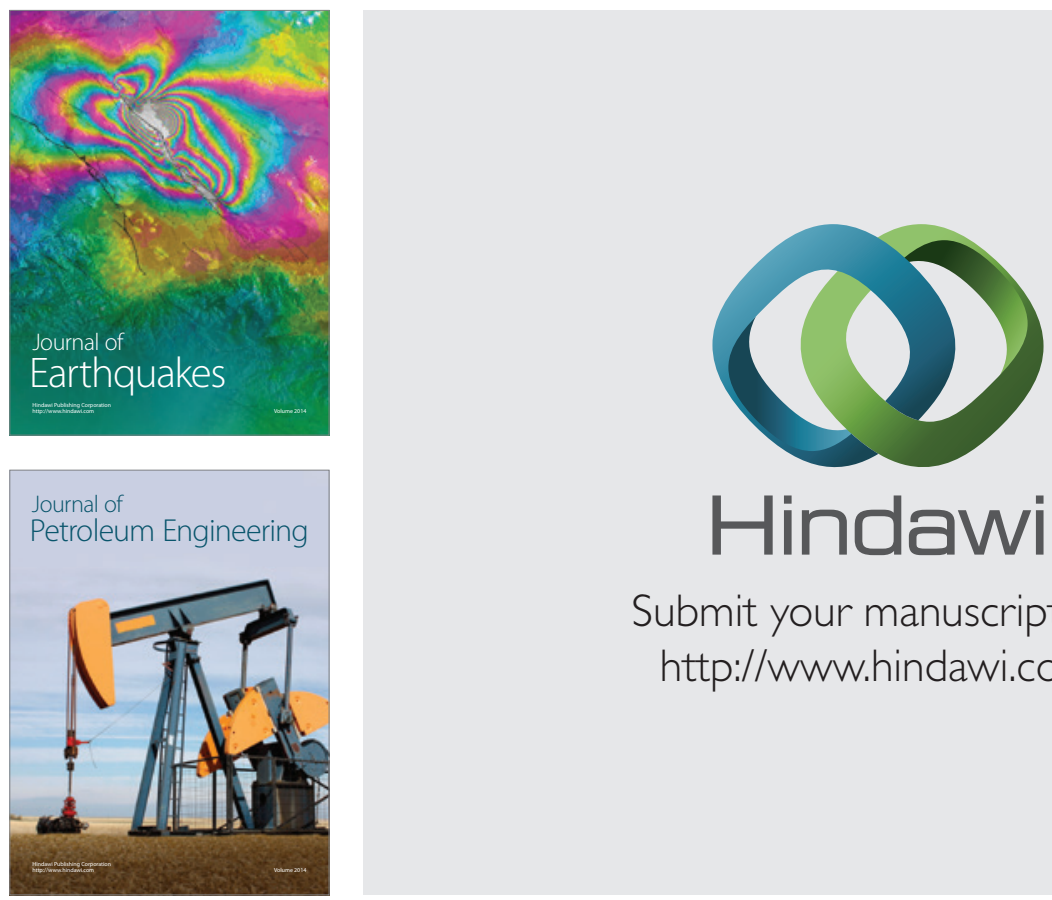

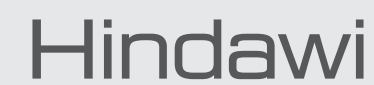

Submit your manuscripts at

http://www.hindawi.com
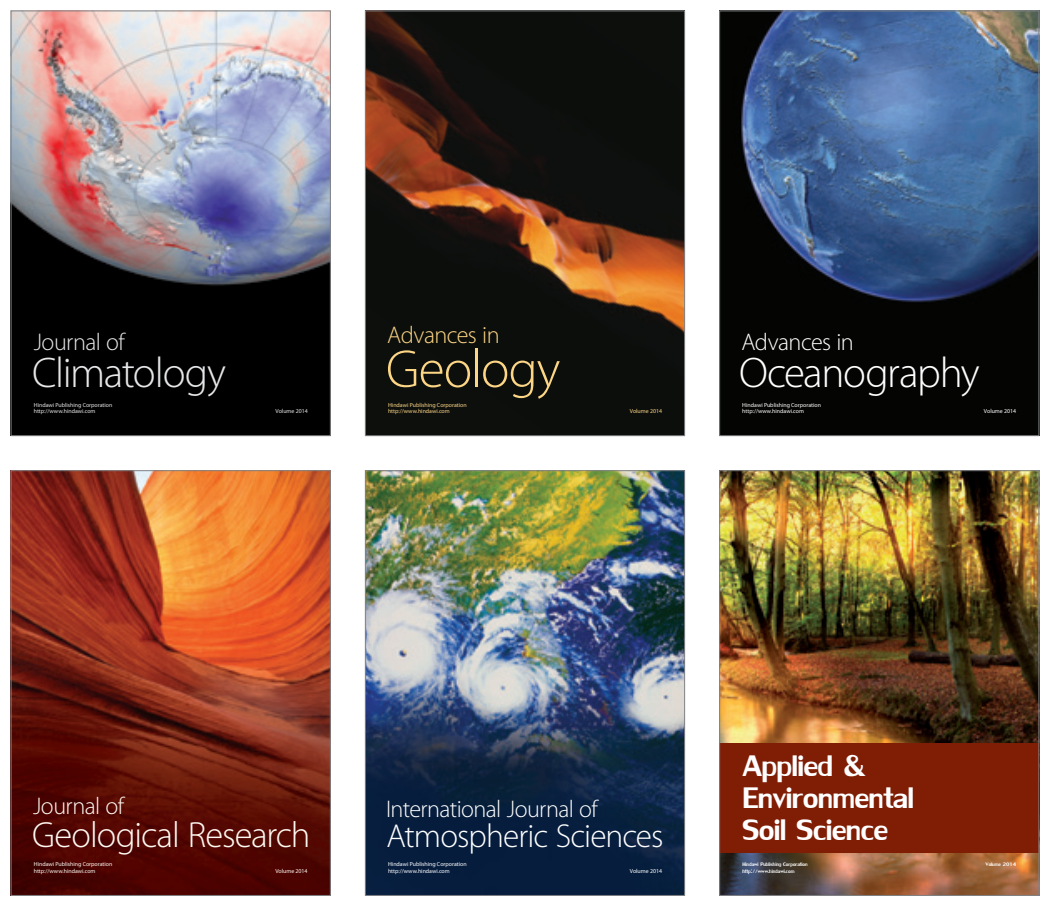
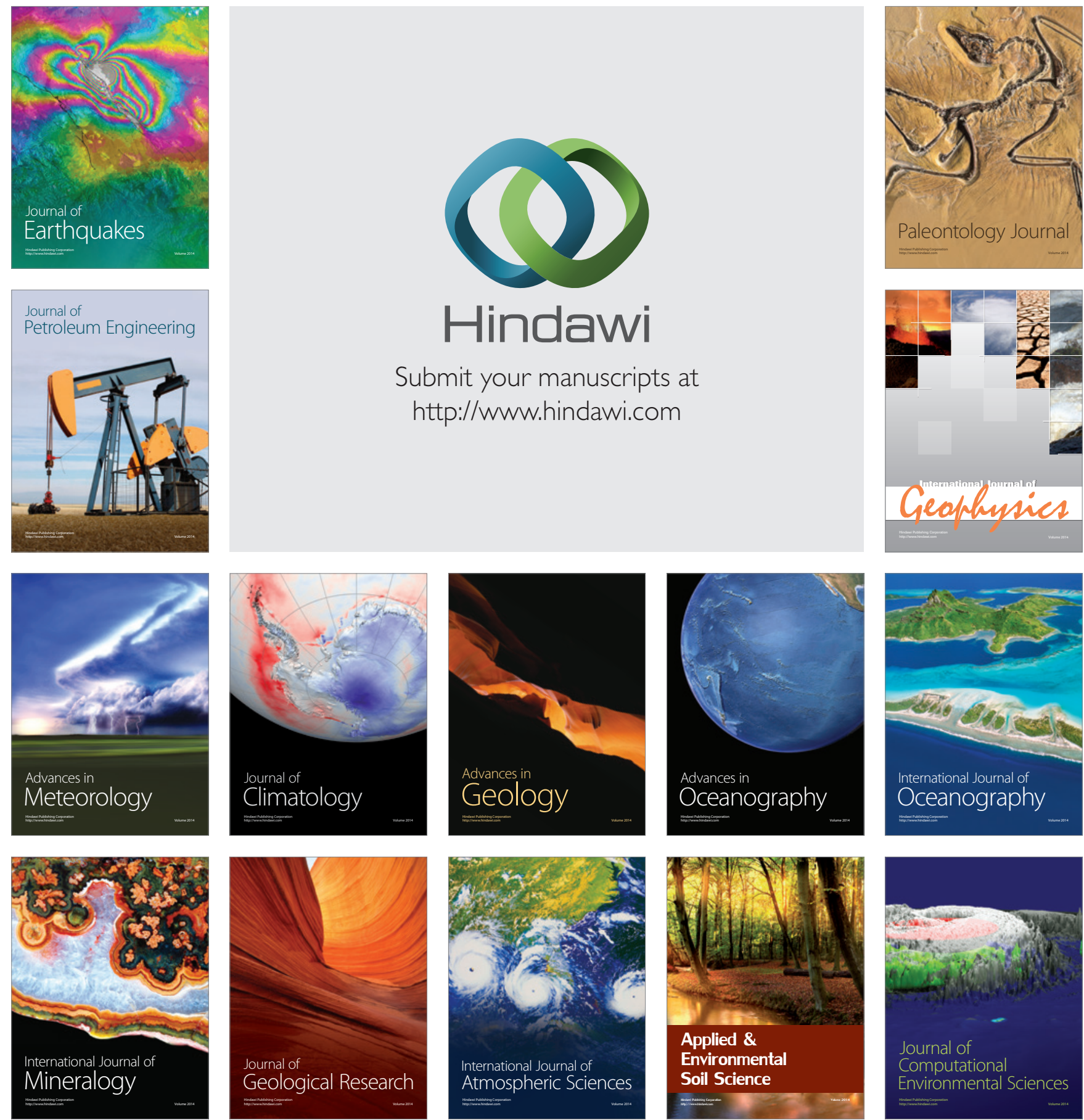